\title{
Functional Outcome after Endoscopic Lumbar Discectomy by Destandau's Technique: A Prospective Study of 614 Patients
}

\author{
Paresh Chandra Dey, Saurav Narayan Nanda \\ Department of Orthopaedics and Trauma, AMRI Hospital, Bhubaneswar, India
}

\begin{abstract}
Study Design: Prospective study.
Purpose: To investigate the efficacy of the lumbar discectomy procedure using the Destandau Endospine System by assessing the functional outcome and complication rate in 614 patients.

Overview of Literature: Endoscopic Spine Surgery (Endospine) using Destandau's technique is an established treatment method of lumbar disk herniation; it was introduced in 1993 and is gaining prominence for its small skin incision and minimal tissue dissection with excellent visualization.

Methods: Of 840 patients, we selected 614 patients, based on the strict inclusion criteria, who underwent endoscopic lumbar discectomy (ELD) between August 2008 and November 2015. The technique comprised localization of the symptomatic level followed by insertion of an endospine system device through a 15-20-mm skin and fascial incision to perform a discectomy. We evaluated results by Macnab's criteria, Oswestry Disability Index (ODI) and Visual Analog Scale (VAS) score after a minimum follow-up of 12 months and maximum up to 54 months.

Results: Based on the modified Macnab's criteria, 479 patients (78.01\%) exhibited excellent, 110 (17.9\%) good, 19 (3.09\%) fair, and $6(0.97 \%$ ) poor results. The average ODI score was improved from 64 (range, 44-86; standard deviation [SD]=9.2) to 14 (range, 2-31; $S D=4.4$ ), and the average VAS score from 7.8 (range, 6-10; $S D=0.9$ ) to 2 (range, $0-7 ; S D=1.2$ ) in a year. On 1-year follow-up, 589 patients $(95.9 \%)$ were completely pain-free and satisfied with the procedure.

Conclusions: ELD provides a safe, effective, and minimal access corridor for lumbar discectomy. The technique also allows early postoperative mobilization and faster return-to-work.
\end{abstract}

Keywords: Endospine; Endoscopy; Diskectomy; Minimally invasive surgical procedures

\section{Introduction}

Low back pain is the leading musculoskeletal condition that affects nearly everyone at some point of time and approximately $4 \%-33 \%$ of the population at any given point
[1]; it is one of the major causes of chronic incapacity in adults aged $<45$ years [2]. Reportedly, $77 \%$ of the population will present with back pain and $35 \%$ with sciatica, at least, once during their lifetime [3], and 10\% of all lumbar disk herniation cases are candidates to surgery [4]. Thus,

Received Dec 7, 2018; Revised Dec 28, 2018; Accepted Feb 20, 2019

Corresponding author: Saurav Narayan Nanda

Department of Orthopaedics and Trauma, AMRI Hospital, Plot No. 1, Beside Satyasai Enclave, Khandagiri, Bhubaneswar - 751030 , India

Tel: +91-0674-666-6600, Fax:+91-0674-666-6600, E-mail: saurav.scb@gmail.com 
low back pain is a major burden of the society and warrants proper attention to provide optimal care to patients by taking help of newer and advanced technologies. Lately, this minimally invasive technique has gained momentum in all areas of surgery, and spinal surgery is no exception.

In most cases, pain because of lumbar disk disease resolves over 6 weeks with the help of anti-inflammatory medication and physiotherapy. However, in more unmanageable cases, epidural steroid injections can assist in pain management [5]. Severe, unremitting pain, neurological deficit, and patients' preference are key indications for surgery. The Main Lumbar Spine Study on sciatica reported that patients with severe symptoms benefited more from surgery than conservative management $(71 \%$ versus 43\%) [6].

Despite various operative procedures available for lumbar disk surgery, from total laminectomy to microdiscectomy, endoscopic discectomy has its own place for its small cosmetic incision, minimal tissue disruption, enhanced visualization because of better magnification and illumination, shorter hospital stay, and faster postoperative recovery time [7]. In 1934, Mixter and Barr [8] first described a surgical procedure to remove a lumbar disk. Later, this technique was improved, which became known as the classical discectomy technique [9]. Mayer and Brock [10] and Foley and Smith [11] described endoscopic discectomy techniques. Of several spinal endoscopic systems used for disk surgery, Destandau Endospine System, Yeung percutaneous endoscopic discectomy, and METRx system are considered viable alternatives to open disk surgery [7] and are performed using standard microsurgical techniques via a minimally invasive approach [12].

This study aims to report a series of 614 patients operated for lumbar discectomy using the Destandau Endospine System at AMRI Hospital, a tertiary heath care centre of Bhubaneswar, India. As most of the existing literature for lumbar discectomy by the Destandau Endospine System has the small sample size, we estimate our study can provide a better picture of effectiveness, safety, and functional outcome of the said procedure.

\section{Materials and Methods}

\section{Patients}

Of 840 patients with different types of lumbar disk herniation at single to multiple levels with radiculopathy were operated between August 2008 and November 2015. Of these, we evaluated 614 patients who fulfilled the following eligibility criteria.

The inclusion criteria were as follows: (1) patients having prolapsed lumbar disc with unilateral radiculopathy; (2) positive 'straight leg raise' (SLR) test or femoral stretch test; (3) magnetic resonance imaging (MRI) findingidentification of a single nerve root lesion; and (4) patients having, at least, 6 weeks of conservative therapy after the appearance of symptoms in the form of bed rest, activity modification, physiotherapy, and medication.

The exclusion criteria were as follows: (1) patients with bilateral radiculopathy; (2) initial first 20 patients in view of the learning curve; (3) multilevel disc prolapse or root involvement; (4) cauda equine syndrome; and (5) patients having uncorrelated clinic radiological features.

We enrolled 614 patients (369 male patients; age, 12-62 years; mean age $=32.4$ years, standard deviation $[\mathrm{SD}]=12.4$ ) in this study, with the mean follow-up of 36.08 months (range, 12-54 months; $\mathrm{SD}=8.9$ ). All patients were operated under general anesthesia and for a single-level disc prolapsed with left radiculopathy in 368 patients and right radiculopathy in the remaining 228 patients. Of 614 patients, 267 patients were operated at L5-S1 level, 328 at L4-L5 level, 15 at L3-L4 level, three at L2-L3 level, and one patient at L1-L2 level. There were 118 extruded, 456 contained, four foraminal, and 46 sequestrated disc herniation cases. We collected detailed preoperative history, clinical examination, radiological details (X-rays and $\mathrm{MRI})$, laboratory tests, consent from all patients undergoing surgery, and intraoperative images and video documentation. The main operating surgeon was the same for all patients. Furthermore, the modified Macnab's criteria, Oswestry Disability Index (ODI) score, and Visual Analog Scale (VAS) score were used to evaluate the results at 24 hours, 1 month, and 1 year postoperatively.

\section{Surgical technique}

All surgeries were performed using the Destandau Endospine System (Karl Storz, Tuttlingen, Germany), which comprises an endospine tube, trocar, and working insert (Fig. 1). The working insert comprises four ports-4$\mathrm{mm}$ endoscope $\left(0^{\circ}\right)$, 4-mm suction cannula, 8 - $\mathrm{mm}$ for the working instrument, and nerve root retractor (Fig. 1). The operative technique comprises modified knee chest positioning (Fig. 2) after the administration of general 

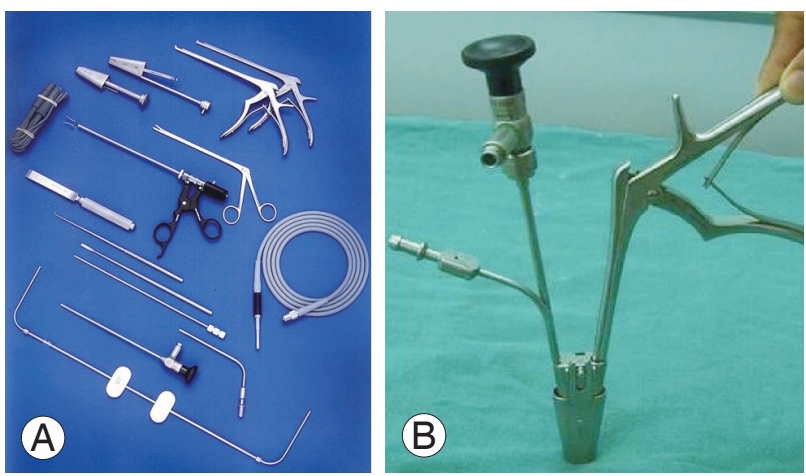

Fig. 1. (A, B) Working instruments with the Destandu Endospine System.

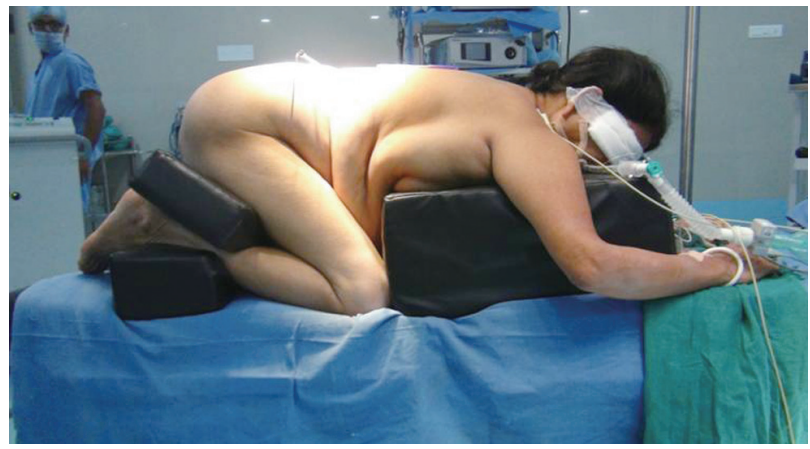

Fig. 2. The modified knee chest positioning.

anesthesia followed by level localization by a localization device (Fig. 3). The skin incision was made at a marked point, $5 \mathrm{~mm}$ away from the midline and 15-20-mm long as required for the body built; aponeurosis was incised using Mayo's scissors. Moreover, 12-mm wide periosteal elevator was used to subperiosteally elevate the paravertebral muscles from the spinous process and superior laminae, thereby exposing the interlaminar window.

The endospine tube with trocar was pushed through the incision in the direction of the localization device over superior laminae followed by the withdrawal of trocar. The working insert was then introduced over the endospine tube; it was locked by the telescoping locking system (Fig. $4)$. Then, the video camera was connected to $0^{\circ} 4-\mathrm{mm}, 18$ $\mathrm{cm}$ rigid endoscope under sterile conditions. The suction tube and endoscope were introduced into their respective ports. Any soft tissue bulging in the mouth of the tube was removed to visualize the interlaminar window (superior and inferior lamina) and facet joint.

Resection of the inferior margin of the superior lamina and excision of yellow ligament was performed leading to the exposure of the dural sac and nerve root. The neuro patties were pushed at the shoulder, either cranial, and cau-
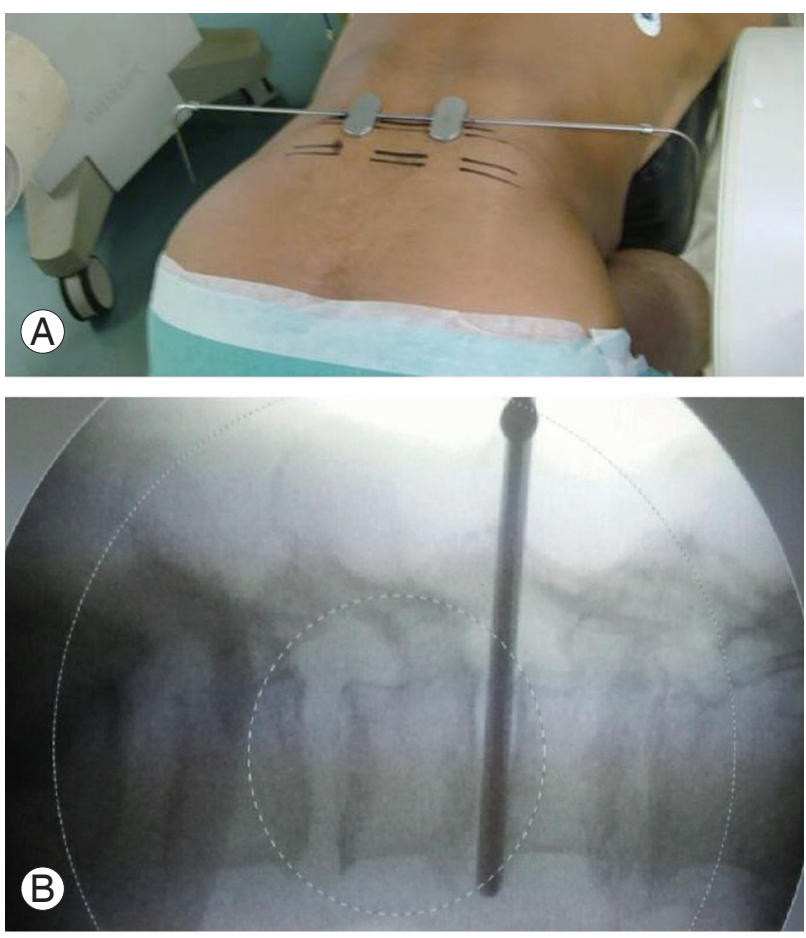

Fig. 3. (A, B) Localizing the device placement and fluoroscopic confirmation.

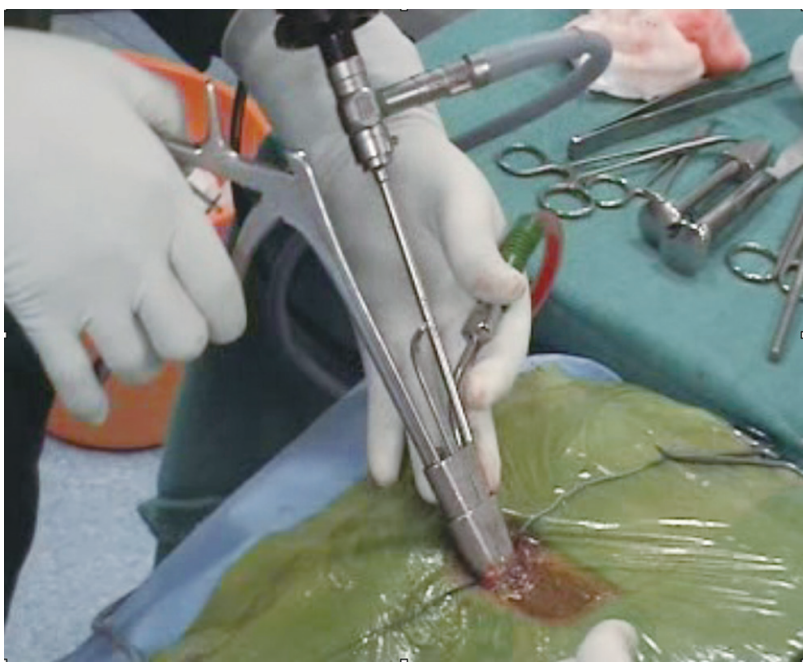

Fig. 4. Introduction of the working instrument.

dal or both directions after the identification of the nerve roots to maintain a bloodless field. The bulged disc was pierced with a spatula, or annulotome, or by 11 no blade. Depending on local findings, discectomy was performed (Fig. 5), and only the loose pieces of the disc were taken out. The disc cavity was irrigated with an isotonic solution and viewed by the telescope to validate adequate nerve root decompression. Then, the nerve root up to the foramina was visualized as adequate and endpoint of decompression. 


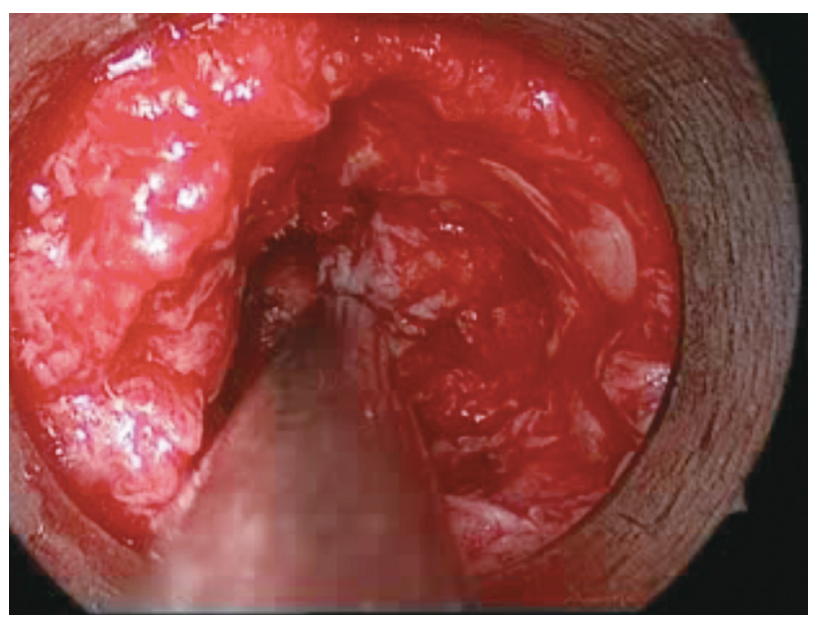

Fig. 5. Procedure discectomy and endoscopic view.

The endospine tube, along with the working insert, was then withdrawn. The cut end of aponeurosis was sutured using 2-0 Vicryl suture followed by closure of the skin with 3-4 vertical subcuticular stitches. Next, a porous dry dressing was applied over the incision. The average operative time was 45 minutes (range, 35-60 minutes), and the average blood loss was $65 \mathrm{~mL}$ (range, $30-80 \mathrm{~mL}$ ).

In central disc herniation, the approach was from the side where the symptoms predominate. In foraminal and extraforaminal disc herniation, the skin incision was slightly higher, muscles dissected a bit more laterally till the lateral limit of the isthmus followed by bone resection up to the base of the pedicle to expose the nerve root and the herniated disc.

All patients were mobilized on the same evening, except eight patients (four patients with a dural tear, two obese with diabetes mellitus, one patient was hypotensive, and one was with severe wound site pain) who were mobilized on the second postoperative day. In addition, 316 patients (51.4\%) were discharged on the same day, 268 (43.6\%) discharged on the first postoperative day, and $30(4.9 \%)$ on the second postoperative day. These patients were followed up on 2 weeks, 6 weeks, 3, 6, 12 months, and subsequent visits. For high-risk and diabetic patients, we evaluated on day 7 to determine after any evidence of infection. Complaints like backache, fever, and leg discomfort were noted accordingly. The SLR was tested on every visit except first. On subsequent visits, all these parameters were evaluated.

\section{Compliance with ethical requirements}

All patients who underwent surgery were explained about

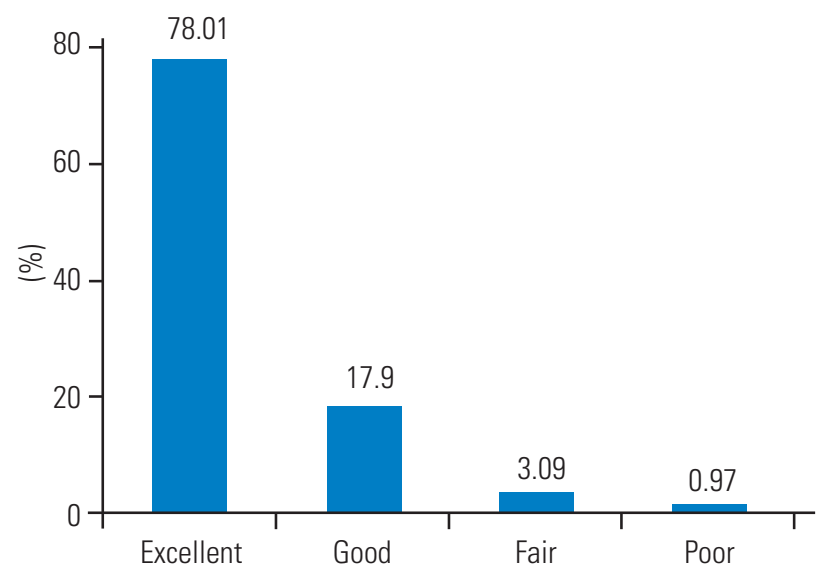

Fig. 6. Macnab's grading.

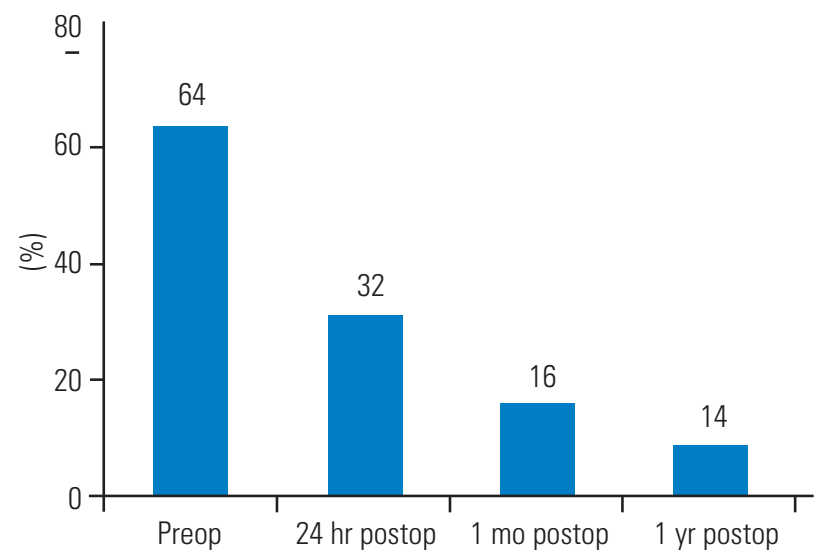

Fig. 7. Oswestry Disability Index score. Preop, preoperative; Postop, postoperative.

the procedure, outcome, and possible adverse effect. Written informed consent was obtained from all patients before the above-described procedure. An approval was obtained from the Ethics Committee of AMRI Hospital, Bhubaneswar, India (IRB approval no., 0162OR).

\section{Results}

On 1-year follow-up, 589 patients (95.9\%) were relieved of sciatica (leg discomfort), completely pain-free, and were satisfied with the procedure. Based on the modified Macnab's criteria, 479 patients (78.01\%) had excellent, 110 (17.9\%) good, 19 (3.09\%) fair, and six (0.97\%) poor results (Fig. 6). The average ODI score was improved from 64 (range, 44-86; $\mathrm{SD}=9.2$ ) to 14 (range, $2-31 ; \mathrm{SD}=4.4$ ) (Fig. 7), and the average VAS score from 7.8 (range, 6-10; $\mathrm{SD}=0.9$ ) to 2 (range, $0-7 ; \mathrm{SD}=1.2$ ) in a year (Fig. 8). 


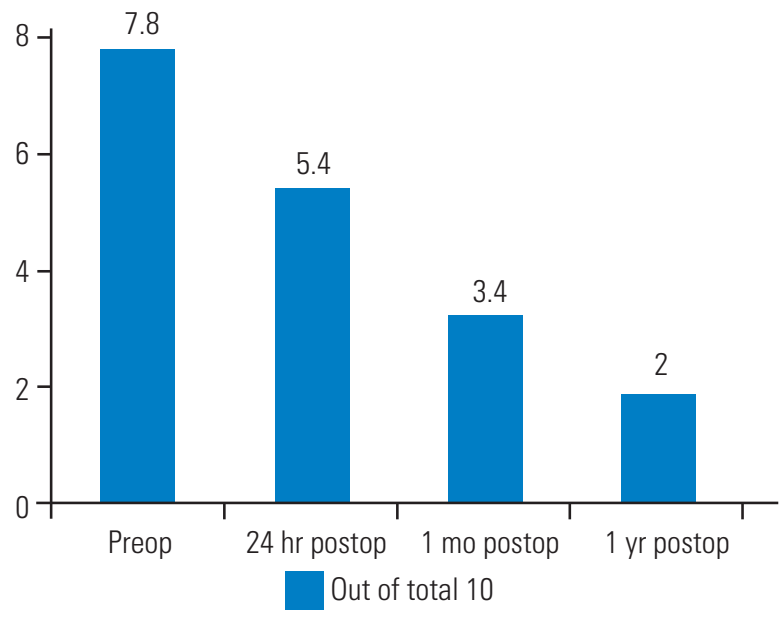

Fig. 8. Visual Analog Scale score. Preop, preoperative; Postop, postoperative.

In addition, 14 patients (2.2\%) had transient paresthesia in the leg, which improved in 2 weeks; one patient $(0.1 \%)$ had transient extensor hallucis longus weakness, which improved in 10 days, four patients $(0.6 \%)$ had wound dehiscence, of whom wound healed in three patients with dressing and local application of supirocin ointment. The remaining one patient needed secondary wound closure and healed in 18 days. Then, four patients $(0.6 \%)$, who had a dural tear and were sealed with muscle patch and surgical treatment, were followed up for any dural leak and discharged on the second postoperative day without any complication. Of note, two patients $(0.3 \%)$ had recurrence of disc in the same level and same side, in one after 2 years 4 months and in another after 3 years and 6 months because of heavy weight lifting; in both cases, disc herniation was confirmed by contrast MRI and operated by the endospine system without any complication with the complete resolution of symptoms at the 6-week follow-up. Superficial wound infection was observed in six patients $(0.9 \%)$, which healed in 15 days by regular dressings, rest, and antibiotics administration. Postsurgical discitis was observed in four patients (0.6\%), which was diagnosed mainly on the clinical ground with raised inflammatory markers and MRI findings. They were treated by intravenous antibiotics (injected piperacillin and tazobactam $4.5 \mathrm{~g} 3$ times daily) for the first week followed by oral antibiotics for 5 weeks. In addition, two patients responded well to antibiotics and no further intervention of any kind was needed. The other two patients had occasional residual backache who were treated by activity modification, analgesics, lumbar support, and rest. In our

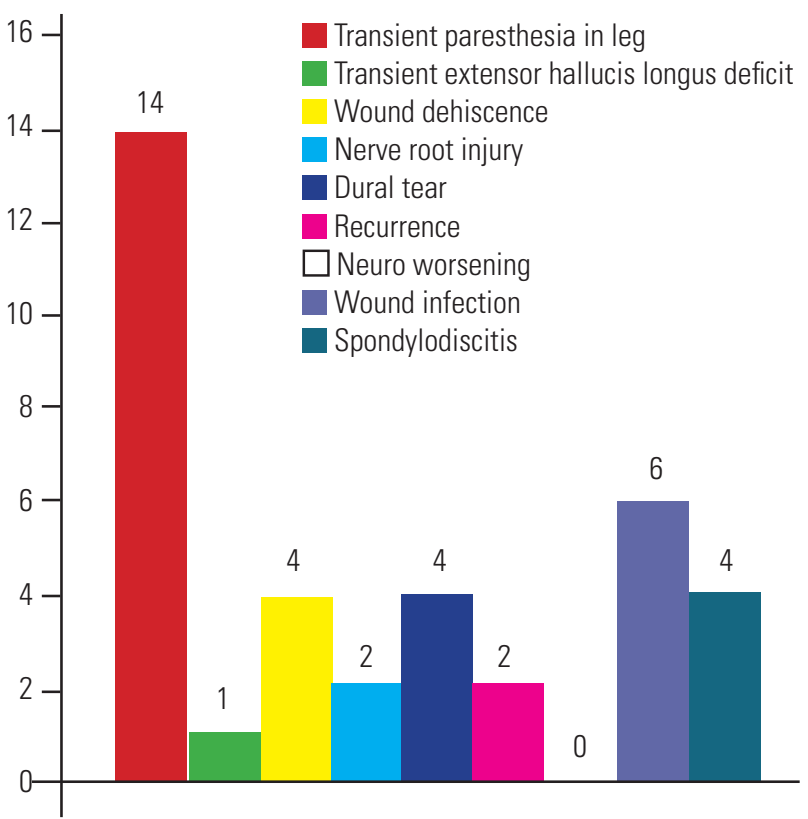

Fig. 9. Complications.

series, we observed partial nerve root injuries in two patients ( $0.3 \%)$; they were encountered by the nerve root retractor while attempting a discectomy at L1-L2 and L2-3 levels. However, nerve roots were only compressed and were in continuity, which improved up to grade 5 lower limb power in 3 months of L2-3 level patients and grade 4 of L1-2 level patients at 6 months (Fig. 9).

\section{Discussion}

Discectomy for lumbar disc herniation is the most commonly performed spinal surgery. The basic principle of different techniques is to relieve the herniation-induced nerve root compression. Mixter and Barr [8] first reported the surgical treatment of patients with lumbar herniated discs by undertaking laminectomy and discectomy. Later, Caspar [13] and Yasargil [14] introduced microscopy into disc surgery, enabling minimal anatomic damage. Typically, the success rates are quite high with all surgical procedures for herniated lumbar disks, amid Williams [15], Ebeling and Reulen [16], Caspar et al. [17], and Chatterjee et al. [18] reporting $73 \%-86 \%$ of success rates. The success rates for microdiscectomy ranged $88 \%-98.5 \%$ in various series [19]. The comparative study of micro- to macrodiscectomy by Katayama et al. [20] reported small statistically significant differences in the operation time, hospitalization, amount of bleeding, and postoperative pain. However, the study established a better postoperative outcome and less morbidity 
with microdiscectomy, resulting in its global acceptance [20].

Other minimally invasive techniques, such as percutaneous lumbar discectomy (manual), as well as automated, chemonucleolysis, and percutaneous laser-assisted discectomy, are intradiscal procedures with their own limitation regarding the orientation of anatomical structures and for noncontained disks and they do not address the concomitant bony and ligamentous compression of the nerve root [19]. The results of these procedures have been highly variable, with satisfactory results $29 \%-92 \%$ [21]. To date, however, no study has illustrated the superiority of these procedures over microsurgical discectomy, which continues to be regarded as the standard with which all other techniques must be compared. Thus, microdiscectomy is considered as the gold standard for lumbar disk surgery [18]. The $95.9 \%$ success rate in this study is also comparable to $90 \%$ excellent to good results reported by Kaushal and Sen [7], 89\% by Łysoń et al. [22], 94\% by Palmer [23] and Perez-Cruet et al. [24], and $92.4 \%$ by Ranjan and Lath [19].

The patients' ability to return to the previous employment is a measure of the success of any surgical procedure. Bookwalter et al. [25] and Caspar et al. [17] reported the mean return-to-work time of 5 and 18.6 weeks, respectively, following microdiscectomy. Palmer [23] reported a mean return-to-work time of 32 days following microdiscectomy, while Perez-Cruet et al. [24] reported the mean return-to-work time of 17 days following microdiscectomy. Our patients returned to previous employment on an average of 15 days with restriction to avoid heavy manual work for 1 month. The advantages of endoscopic discectomy over standard microdiscectomy include smaller incision, improved intraoperative visualization, lesser postoperative pain, early postoperative ambulation, shorter hospital stay, and shorter return-to-work time.

The complications reported in a large series with patients undergoing microdiscectomy included wound infections $(0 \%-7.2 \%)$, discitis $(0 \%-0.8 \%)$, dural tears $(0 \%-$ $6.7 \%)$, and recurrent disc prolapse $(3 \%-14 \%)$ [19]. The complications observed in our series are comparable to other microdiscectomy series. In this series, we noted $0.6 \%$ cases of discitis, $0.6 \%$ of dural injury, and $0.3 \%$ of nerve root injury. Destandau [26], Perez-Cruet et al. [24], and Ranjan and Lath [19] had average surgical time of $66 \mathrm{~min}$ utes, average blood loss of $22 \mathrm{~mL}$, average hospital stay of 7.7 hours, complication rate of $5 \%$, reoperation rate of $4 \%$, and average return-to-work time of 17 days with satisfac- tory results in $94 \%$ of patients. Our series had the average operative time of 45 minutes (range, 39-53 minutes; $\mathrm{SD}=4.1$ ), average blood loss of $65 \mathrm{~mL}$ (range, $30-80 \mathrm{~mL}$; $\mathrm{SD}=11.9$ ), and average hospital stay of 16.2 hours (range, $8-48$ hours; $\mathrm{SD}=8.4$ ). The complication rate was $6.0 \%$ (total 37 patients), reoperation rate was $0.4 \%$ (three patients, two patients for recurrent disc, and one for superficial infection debridement). The average return-to-work time was 15 days (range, 7-28 days; $\mathrm{SD}=4.4$ ), and overall results were $95.9 \%$ (589 patients), which are comparable.

This case series (614 patients) establishes that endoscopic lumbar discectomy (ELD) is one of the accepted surgical procedures that provides a safe, effective, and minimal access operative technique for lumbar discectomy. The technique also enables early postoperative mobilization and faster return-to-work time with cosmetic scar mark. The limitations of this study are that the procedure needs expertise, a learning curve, and instrumentations. However, according to our experience, it can be very well reproducible with a short span of training and practice.

\section{Conclusions}

Compared with the existing literature, ELD is a safe and effective procedure to address lumbar disc herniation surgically. It is now an established technique with advantages of minimal skin and soft tissue handling, magnified intraoperative images and postoperative cosmetic surgical scar. In addition, the technique allows early postoperative mobilization and faster return-to-work. Our results, as well as those from other authors, are highly favorable and encouraging, and we believe that in a few years, the endoscopic approach will become the new 'gold standard' for lumbar disc surgery. Hence, we recommend ELD in properly selected patients.

\section{Conflict of Interest}

No potential conflict of interest relevant to this article was reported.

\section{References}

1. Woolf AD, Pfleger B. Burden of major musculoskeletal conditions. Bull World Health Organ 2003;81:64656. 
2. Gibson JN, Waddell G. Surgical interventions for lumbar disc prolapse. Cochrane Database Syst Rev 2007:CD001350.

3. Gotfryd A, Avanzi O. A systematic review of randomised clinical trials using posterior discectomy to treat lumbar disc herniations. Int Orthop 2009;33:117.

4. Ozer AF, Keskin F, Oktenoglu T, et al. A novel approach to the surgical treatment of lumbar disc herniations: indications of simple discectomy and posterior transpedicular dynamic stabilization based on carragee classification. Adv Orthop 2013;2013:270565.

5. Chou R, Qaseem A, Snow V, et al. Diagnosis and treatment of low back pain: a joint clinical practice guideline from the American College of Physicians and the American Pain Society. Ann Intern Med 2007;147:478-91.

6. Atlas SJ, Deyo RA, Keller RB, et al. The Maine Lumbar Spine Study, part II: 1-year outcomes of surgical and nonsurgical management of sciatica. Spine (Phila Pa 1976) 1996;21:1777-86.

7. Kaushal M, Sen R. Posterior endoscopic discectomy: results in 300 patients. Indian J Orthop 2012;46:81-5.

8. Mixter WJ, Barr JS. Rupture of intervertebral disc with involvement of spinal canal. New Engl J Med 1934;211:210-5.

9. Gotfryd A, Avanzi O. A systematic review of randomised clinical trials using posterior discectomy to treat lumbar disc herniations. Int Orthop 2009;33:117.

10. Mayer HM, Brock M. Percutaneous endoscopic discectomy: surgical technique and preliminary results compared to microsurgical discectomy. J Neurosurg 1993;78:216-25.

11. Foley KT, Smith MM. Microendoscopic discectomy. Tech Neurosurg 1997;4:301-7.

12. Ozturk C, Tezer M, Aydogan M, Sarier M, Hamzaoglu A. Posterior endoscopic discectomy for the treatment of lumbar disc herniation. Acta Orthop Belg 2006;72:347-52.

13. Caspar W. A new surgical procedure for lumbar disc herniation causing less tissue damage through a microsurgical approach. In: Wullenweber R, Brock M, Hamer J, Klinger M, Spoerri O, editors. Lumbar disc adult hydrocephalus. Berlin: Springer Berlin Heidelberg; 1977. p. 74-80.
14. Yasargil MG. Microsurgical operation of herniated lumbar disc. In: Wullenweber R, Brock M, Hamer J, Klinger M, Spoerri O, editors. Lumbar disc adult hydrocephalus. Berlin: Springer Berlin Heidelberg; 1977. p. 81.

15. Williams RW. Microlumbar discectomy: a conservative surgical approach to the virgin herniated lumbar disc. Spine (Phila Pa 1976) 1978;3:175-82.

16. Ebeling U, Reulen HJ. Are there typical localisations of lumbar disc herniations?: a prospective study. Acta Neurochir (Wien) 1992;117:143-8.

17. Caspar W, Campbell B, Barbier DD, Kretschmmer $\mathrm{R}$, Gotfried Y. The Caspar microsurgical discectomy and comparison with a conventional standard lumbar disc procedure. Neurosurgery 1991;28:78-86.

18. Chatterjee S, Foy PM, Findlay GF. Report of a controlled clinical trial comparing automated percutaneous lumbar discectomy and microdiscectomy in the treatment of contained lumbar disc herniation. Spine (Phila Pa 1976) 1995;20:734-8.

19. Ranjan A, Lath R. Microendoscopic discectomy for prolapsed lumbar intervertebral disc. Neurol India 2006;54:190-4.

20. Katayama Y, Matsuyama Y, Yoshihara H, et al. Comparison of surgical outcomes between macro discectomy and micro discectomy for lumbar disc herniation: a prospective randomized study with surgery performed by the same spine surgeon. J Spinal Disord Tech 2006;19:344-7.

21. Maroon JC. Current concepts in minimally invasive discectomy. Neurosurgery 2002;51:S137-45.

22. Lyson T, Mariak Z, Jadeszko M, Kochanowicz J, Lewko J. Results of Destandau microendoscopic lumbar discectomy. Neurol Neurochir Pol 2008;42:10511.

23. Palmer S. Use of a tubular retractor system in microscopic lumbar discectomy: 1 year prospective results in 135 patients. Neurosurg Focus 2002;13:E5.

24. Perez-Cruet MJ, Foley KT, Isaacs RE, et al. Microendoscopic lumbar discectomy: technical note. Neurosurgery 2002;51:S129-36.

25. Bookwalter JW 3rd, Busch MD, Nicely D. Ambulatory surgery is safe and effective in radicular disc disease. Spine (Phila Pa 1976) 1994;19:526-30.

26. Destandau J. Technical features of endoscopic surgery for lumbar disc herniation: 191 patients. Neurochirurgie 2004;50:6-10. 